\title{
A Thermodynamic Based Plant-Wide Control Design Procedure of the Tennessee Eastman Process
}

\author{
Luis T. Antelo, Irene Otero-Muras, Julio R. Banga and Antonio A. Alonso \\ Process Engineering Group. IIM-CSIC. C/ Eduardo Cabello, 6. 36208-VIGO (Spain)
}

\begin{abstract}
In this work, we apply the systematic approach to plant-wide control design presented in [1], based on the fundamentals of process networks, thermodynamics and systems theory, to the Tennessee Eastman (TE) Challenge Process, deriving robust decentralized controllers that will ensure the stability of the complete plant. We take one step further in the control design procedure by completing it with the realization of the controllers. The inventory control laws proposed are derived from a set of control loops over the available degrees of freedom in the process.
\end{abstract}

Keywords: Thermodynamics, Plant-Wide Control Design, Process Networks, Inventory Control, Control Realization.

\section{Introduction}

The TE process defined in [2] produces two products $(G$ and $H$ ) from four reactants $(A$, $C, D$ and $E$ ). A further inert trace component $(B)$ and one byproduct $(F)$ are present. The process consists of a continuous stirred tank reactor, a condenser, a flash drum and a stripper. In Figure 1, the flowsheet of the TEP is reproduced. The gaseous reactants are fed to the reactor where they are transformed into liquid products. The following reactions take place in gas phase:

$$
\begin{aligned}
\mathrm{A}_{(\mathrm{g})}+\mathrm{C}_{(\mathrm{g})}+\mathrm{D}_{(\mathrm{g})} & \rightarrow \mathrm{G}_{(\mathrm{l})} \\
\mathrm{A}_{(\mathrm{g})}+\mathrm{C}_{(\mathrm{g})}+\mathrm{E}_{(\mathrm{g})} & \rightarrow \mathrm{H}_{(\mathrm{l})} \\
\mathrm{A}_{(\mathrm{g})}+\mathrm{E}_{(\mathrm{g})} & \rightarrow \mathrm{H}_{(\mathrm{l})} \\
3 \mathrm{D}_{(\mathrm{g})} & \rightarrow 2 \mathrm{~F}_{(\mathrm{l})}
\end{aligned}
$$

These reactions are irreversible and exothermic with rates that depend on temperature through Arrhenius expressions and on the reactor gas phase concentration of the reactants. The reaction heat is removed from the reactor by a cooling bundle. The products and the unreacted feeds pass through a cooler and, once condensed, they enter a vapor-liquid separator. The noncondensed components recycle back to the reactor feed and the condensed ones go to a product stripper in order to remove remaining reactants by stripping with feed stream. Products $\mathrm{G}$ and $\mathrm{H}$ are obtained in bottoms. The inert (B) and the byproduct (F) are mainly purged from the system as a vapor from the vaporliquid separator.

Since the publication of the TE process example, it has been widely used in the literature as a case study due to its challenging properties from a control engineering 
point of view: it is highly nonlinear, open-loop unstable and it presents a large number

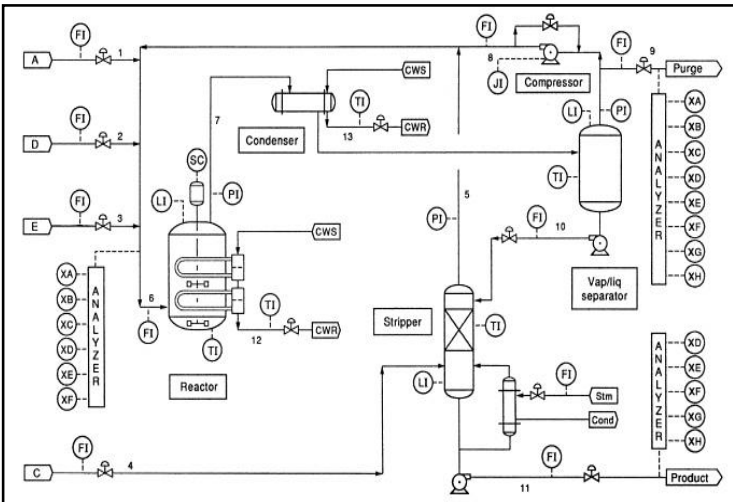

Figure 1: The Tennessee Eastman Process of measured and manipulated variables what offer a widely set of candidates of possible control strategies. A review of the most relevant plant-wide control strategies applied to the $\mathrm{TE}$ process is given in [3] and references there in. Many of the previous proposed approaches have weaknesses as described in [3]. Our contribution try to overcome these drawbacks by systematizing the development of robust decentralized controllers that will ensure the TEP stability. In this framework, the TEP is represented as a process network and mass and energy inventory control loops for each node are designed first to guarantee that the states of the plant will remain on a convex invariant region. In these sets, the second law of thermodynamics provides us with a function -the entropy of the system- which has a definite curvature (is concave). In addition, it can be proved that in these compact regions, the system will be passive and, therefore, stable. At this point, the next step will be the controller realization using the physical inputs-outputs of the process. In the real TE process, inventory fluxes can be the result of combining multiple convective mass or energy flows and the inventory control law has to be obtained as a composition of control loops implemented over the real manipulated variables available in the process.

The paper is structured as follows: In Section 2, network fundamentals and the TEP network representation are described. The thermodynamic formalism used in the design of decentralized controllers is presented in Section 3. Finally, in Section 4 we show the realization of the inventory controllers and we validate the proposed methodology with simulation results.

\section{The Tennessee Eastman Process Network}

As presented in a previous work [1], a process network is defined by a number $j=1, \ldots$, $n$ of well mixed homogeneous material regions connected by material and energy fluxes we will refer to as nodes, plus an extra region $j=0$ which represents the environment. To each node $j$ in the network we associate a state vector $z_{j} \in \mathbb{R}^{c+1}$ of the form $z_{j}=\left(n_{i}^{j}, \ldots, n_{c}^{j}, u^{j}\right)^{T}$, where $n_{i}^{j}$ denotes the mole numbers of component $i$, the internal energy is represented by $u^{j}$ and $c$ stands for the total number of chemical species. With this, the inventory network dynamics can be represented by:

$\dot{m}_{l}=N \phi_{l} ; \dot{u}_{l}=N p_{l} \quad$ with $\quad \dot{m}_{l}, \dot{u}_{l}, \phi_{I}, p_{l} \in \mathbb{R}^{+\ell}$ 
where $\phi_{l}=\sum_{i \in D} \sum_{k=1}^{c} \sigma^{k} f_{i}^{k}$ and $p_{I}=\sum_{i \in D} p_{i}$ are the mass and energy inventory flows,

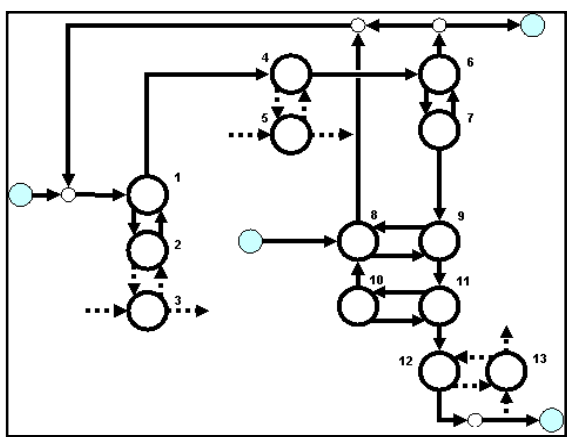

Figure 2: The Tennesse Eastman Network respectively and $N \in \mathbb{R}^{\ell \times \ell}$ is, by definition, a column conservation matrix. In addition, $\ell$ represents the number of dissipative subnetworks (defined as those only interconnected by dissipative fluxes) in which the original system can be simplified and $\boldsymbol{D}$ is the number of nodes forming each dissipative subnetwork. For further information about this network formalism, see [1] and [4]. The network description of the model is depicted in Figure 2 and derives from the TEP flowsheet presented in Figure 1 by assigning one node to each phase. There, the mass and energy flows are represented by solid and dashed arrows, respectively, and the solid circles symbolize the environment. By applying the inventory network concepts presented, the inventory dynamics can be written as:

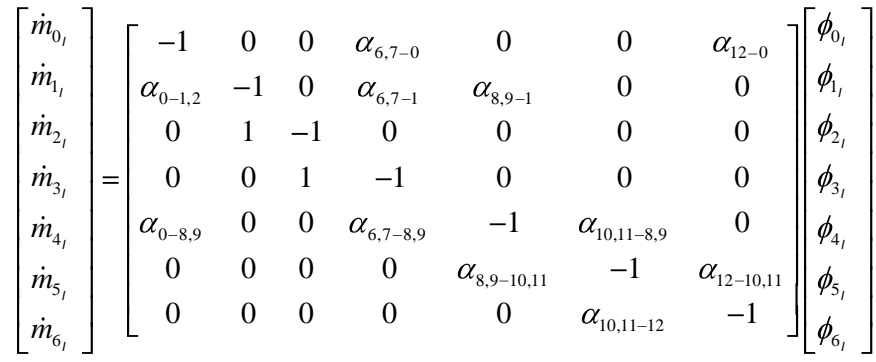

where $\alpha_{i, j}$ represents the fraction of the inventory flow going from node $i$ to node $j$. The resulting inventory network is as depicted in Figure 3. In a similar way, we can represent the energy inventory network (Figure 4), where the main difference with the mass network is that there exist more connections with node 0 (inputs from the environment corresponding to coolant and steam flows in the condenser before the LV separator and the stripper). The underlying structures presented in Figures 3 and 4 for mass and energy inventory layers are the basis of the hierarchical control process design presented next.

\section{Thermodynamic basis for decentralized controller design}

In every node of a process network of volume $v_{\mathrm{j}}$, thermodynamics provides us with a continuous, twice differentiable scalar function $S_{j} z_{j}, v_{j}: \mathbb{R}^{+c+2} \mapsto \mathbb{R}$ named entropy. This function is first order homogeneous in all their arguments and strictly concave with respect to the vector $z_{j}$ [1]. Such property indicates that $S$ has a definite curvature, ensuring that over the set 


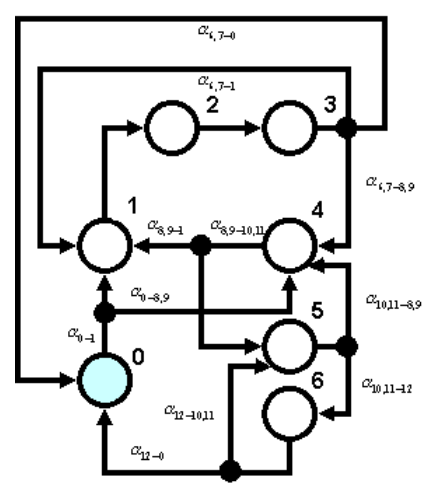

Figure 3: Mass TEP Fundamental Network

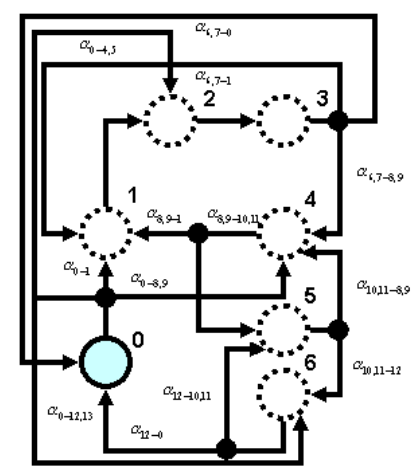

Figure 4: Energy TEP Inventory Network

$\Lambda D=\left\{\left(z_{j}, v_{j}\right)_{j=1}^{n} \mid \quad \sum_{j \in D} v_{j}=v ; \sum_{j \in D} u_{j}=u ; \sum_{j \in D} \sum_{k=1}^{c} \sigma^{k} n_{j}^{k}=m\right\}$

has a maximum for a given $(m, u, v)$ constant and for every $j \in D$. In the last expression, $\sigma^{k}$ represents the vector of molecular weights for the $c$ components. Once the network states converge to $\Lambda$, we can ensure that the process system is passive and, therefore, stable [5]. This fact motivates a hierarchical control design decomposition in which mass and energy inventory controllers in every node of the network are first designed to drive the system to this compact set $\Lambda$. In particular, the mass and energy inventory control layers consist of linear proportional controllers of the form:

$\phi_{I}=\phi_{I}^{*}+\omega_{m} m_{l}-m_{l}^{*} ; p_{l}=p_{l}^{*}+\omega_{u} u_{l}-u_{l}^{*}$

We select a $\phi_{l}{ }^{*} \in \operatorname{Null}(N)$, i.e. $N \phi_{l}{ }^{*}=0$ and $\omega_{n}, \omega_{u}$ are appropriate gain matrices constructed in a way such that the real part of the eigenvalues associated to $N \omega_{m}$ and $N \omega_{u}$

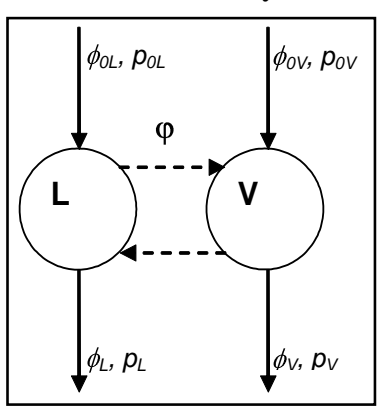
are negative. Finally, taking deviation variables with respect a given reference and substituting the expressions in (7) into (5), it follows that $m_{l} \rightarrow m_{l}{ }^{*}$ and $u_{l} \rightarrow u_{l}{ }^{*}$ exponentially fast so the convergence of the process states to the set $\Lambda$ is ensured. For the TE inventory networks represented in Figures 3 and 4, there exist enough degrees of freedom (DOF) to implement the mass inventory control, using the total inventory outflow of each node as the manipulated variables. For the energy layer, the additional connections with node 0 provides extra DOF to

Figure 5: Dissipative network control this energy inventory. It must pointed out that, as demonstrated in [1], control laws represented in Eqn. (7) do not prevent the system from exhibit complex behavior, such as multiplicities, due to the fact that the convergence of the intensive variables to a unique stable point can not be guaranteed. As presented before, the network entropy is strictly concave in $\Lambda$ and intensive variable control can be designed by, for instance, methods discussed in [5]. In order to avoid these problems and ensure global stability for the TE plant, we propose additional control loops for 
mass and energy inventories in every node belonging to the dissipative subnetworks as depicted in Figure 5. For instance, the mass inventory of a vapor phase is related with the pressure and together with the energy inventory -that is related with the temperature, the composition of equilibrium systems can be modified through these inventories. In order to develop these extra control loops, we consider that the control laws in Eqns. (7) can be obtained as a composition of the control laws applied to each node in D. In Figure 5 , two possible manipulated variables (the outflows of each node) are proposed. Using control laws for the mass inventories in each node analogous to (7):

$\phi_{L}=\phi_{L}^{*}+\omega_{L} \quad m_{L}-m_{L}^{*} ; \phi_{V}=\phi_{V}^{*}+\omega_{V} \quad m_{V}-m_{V}^{*}$

It follows that the sum of equations in (8) results in $\phi_{l}=\phi_{l}^{*}+\omega m_{l}-m_{l}^{*}$ for $\omega_{L}=\omega_{V}$, ensuring convergence of both total and node mass inventories. The formalism concerning the inventory control laws must then be translated into suitable control loops using the available inputs-outputs of the system. This question will be discussed in the next section.

\section{Realization of the Hierarchical Control Design for the TE Process}

Starting from the fundamental process network showed in Figure 2, inventory control loops are developed for every node, except for the condenser and for the nodes representing heat sources or sinks in the energy layer, where there is no mass holdup. The manipulated variables will be the outflows of each node and the flows from node 0 for the mass and energy cases, respectively. Then, the resulting mass and energy

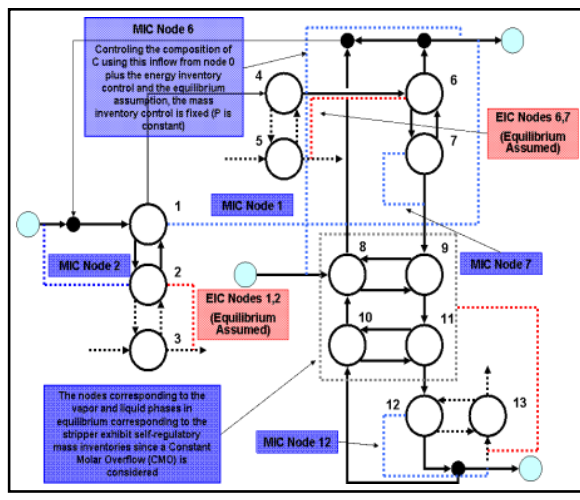

Figure 6: Inventory Control Loops inventory control structures (MIC and EIC, respectively) for the $\mathrm{TE}$ are presented in Figure 6. Note that for mass inventory in node 1 , we do not use the outflow since in the TE there are not valves neither in the stream leaving the reactor nor in the stream from the condenser to the LV separator. Therefore, the next vapor stream (purge) is used. In addition, for every dissipative subnetwork, the equilibrium condition is assumed, which implies $\mu_{i}=\mu_{j} ; T_{i}=T_{j}$ and $P_{i}=P_{j}$ for all $i, j \in D$. This allows us to define a unique energy inventory control loop for each dissipative subnetwork. For the case of node 6 representing the vapor phase in the LV separator, a composition control as depicted in Figure 6 is considered. Since $T_{6}, V$ and $x_{6}$ are being controlled and the nodes are in equilibrium, the pressure as well as the mass inventory are fixed. Finally, the mass inventories for the dissipative subnetworks representing the equilibrium trays of the stripper will be constant, under the assumption of a constant molar overflow (CMO) model. The dynamic performance of the proposed control structure against changes in the A/C Feed ratio (IDV1) is presented in Figure 8, 
showing stability and fast convergence of the process variables to their reference values. Similar results were obtained against other disturbances. Note that it is possible to tune the controllers solving an optimization problem (minimizing the cost function presented in [2]).

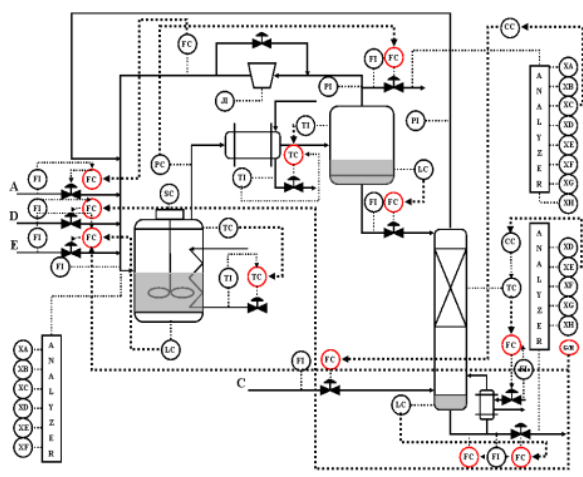

Figure 7: Proposed Control Structure for TEP

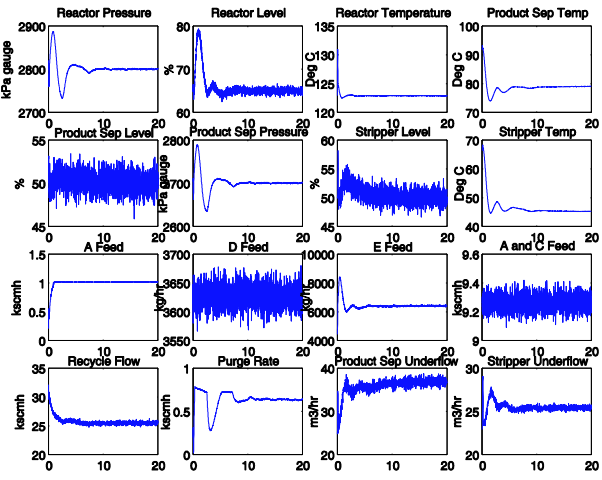

Figure 8: Dynamic Response against IDV(1)

\section{Conclusions}

In this contribution, the systematic plant-wide control design methodology presented in [1] has been applied to the challenging benchmark of the Tennessee Eatman Process. We have designed decentralized control structures which simultaneously ensure stabilization of both plant extensive and intensive variables, paying special attention in the realization of the controllers by making use of the available manipulated variables of the process. The proposed control structure has been tested dynamically with good stability results.

\section{References}

[1]. Antelo, L. T., Otero-Muras, I., Banga, J.R., Alonso, A. A. (2005). A systematic approach to plant-wide control based on thermodynamics. $15^{\text {th }}$ European Symposium on Computer Aided Chemical Engineering (ESCAPE-15), 20(B), 1105-1110.

[2]. Downs, J.J., Vogel, E.F. (1993). A plant-wide industrial process control problem. Computers \& Chemical Engineering, 17, 245-255.

[3]. Larsson, T., Skogestad, S. (2000). Plantwide control: A review and a new design procedure. Modeling, Identification and Control, 21, 209-240.

[4]. Antelo, L. T., Otero-Muras, I., Banga, J.R., Alonso, A. A. (2005). A systematic approach to plant-wide control based on thermodynamics. Submitted to Computers \& Chemical Engineering. [5]. Alonso, A.A., B.E. Ydstie, 2001, Stabilization of distributed systems using irreversible thermodynamics. Automatica, 37, 1739.

\section{Acknowledgements}

The authors acknowledge the financial support received from the Spanish Government (MCyT Projects PPQ2001-3643) and Xunta de Galicia (PGIDIT02-PXIC40209PN). 